\title{
Calibration of the MIRAS Radiometers
}

\author{
Ignasi Corbella (), Francesc Torres, Senior Member, IEEE, Nuria Duffo, Senior Member, IEEE, Israel Durán, \\ Verónica González-Gambau, Roger Oliva, Member, IEEE, Josep Closa, \\ and Manuel Martín-Neira ${ }^{\circledR}$, Senior Member, IEEE
}

\begin{abstract}
The microwave imaging radiometer with aperture synthesis (MIRAS) is formed by 69 total power radiometers, of which three are the noise-injection type. Their calibration is reviewed on the basis of the data gathered during more than eight years of operation. Internally calibrated gain and offset corrections with improved temporal stability are presented. New front-end loss characterization with lower seasonal dependence originated from external temperature swings is also proposed. Finally, a methodology to validate the external calibrations, with the instrument pointing to the cold sky, is developed. It seems to indicate that the change of orientation of the instrument, with associated thermal variations, may induce small changes in the radiometer front-end losses, thus introducing calibration errors.
\end{abstract}

Index Terms-Interferometric synthetic aperture radiometry, L-band radiometry, radiometer calibration, Soil Moisture and Ocean Salinity (SMOS).

\section{INTRODUCTION}

$\mathbf{M}$ ICROWAVE imaging radiometer with aperture synthesis (MIRAS) is an L-band Y-shape two-dimensional (2-D) interferometric radiometer [1] installed on board the European Space Agency satellite Soil Moisture and Ocean Salinity (SMOS), launched in November 2009 with the scientific objective of measuring global soil moisture and ocean salinity [2], [3]. Since mid 2010, MIRAS has been continuously providing fullpolarimetric brightness temperature maps of the Earth surface at a rate of one complete image of the four Stokes parameters

Manuscript received June 28, 2018; revised October 29, 2018; accepted November 27, 2018. This work was supported in part by the European Space Agency and Deimos Enginheria (Portugal) in SMOS P7 Subcontract DME CP12 no. 2015-005, and in part by Spanish public funds, projects TEC2014-58582-R, TEC2017-88850-R, and ESP2015-67549-C3-1-R. (Corresponding author: Ignasi Corbella.)

I. Corbella, F. Torres, N. Duffo, and I. Durán are with the Remote Sensing Laboratory, Universitat Politécnica de Catalunya, 08034 Barcelona, Spain (e-mail: corbella@tsc.upc.edu; xtorres@tsc.upc.edu; duffo@tsc.upc.edu; israel. duran@tsc.upc.edu).

V. González-Gambau is with the Department of Physical Oceanography and Barcelona Expert Center, Institute of Marine Sciences (ICM-CSIC), 08003 Barcelona, Spain (e-mail: vgonzalez@icm.csic.es).

R. Oliva is with the European Space Astronomy Centre, European Space Agency, 28692 Madrid, Spain (e-mail: Roger.Oliva.Balague@esa.int).

J. Closa is with the Space Division, Airbus Defence, 404-28022 Madrid, Spain (e-mail: josep.closa@airbus.com).

M. Martín-Neira is with the European Space Research and Technology Centre, European Space Agency, 2200 AG Noordwijk, The Netherlands (e-mail: Manuel.Martin-Neira@esa.int).

Color versions of one or more of the figures in this paper are available online at http://ieeexplore.ieee.org.

Digital Object Identifier 10.1109/JSTARS.2018.2885576 every $2.4 \mathrm{~s}$. A comprehensive review of the instrument performance and calibration after six years in orbit, including, a fairly complete list of references, can be found in [4].

The instrument has 69 dual polarization small antennas distributed along a Y-shaped structure. Three of them, located near the center of the array, are connected to three corresponding full polarimetric noise injection radiometers (NIR). The other 66 are connected to low-noise receivers to measure both polarizations sequentially by means of switches. Full polarimetric operation of the whole instrument is achieved thanks to a smart use of these switches [5].

At each snapshot, brightness temperature maps are synthesized by image reconstruction of the visibility function [6], [7] For any two different antennas, the visibility is measured by cross correlating their output signals, after amplification and filtering, using an array of one-bit two-level digital correlators [3] insensitive to the signal amplitudes, and thus, providing only the normalized value of correlation products [8]. Denormalized amplitude is obtained by measuring the system temperature using a power measurement system (PMS) available at each receiver [9].

The visibility at zero spacing is the antenna temperature and can be measured by any single receiver. Three NIRs are installed near the center of the array for this purpose. Initially, the average of the three measurements was used, but after some time, it was decided to keep only one of the three NIR units due to relatively large temporal instabilities shown by the other two.

The zero-spacing visibility can also be set equal to the average antenna temperatures measured by all (or some) individual MIRAS receivers (called LICEF for lightweight cost-effective front end), each one acting as a total power radiometer. This technique provides fairly stable data in long-term trends [10], [11] but it is still too sensitive to random jumps often observed in diode-detected output voltages. This option is not yet considered as baseline for the SMOS processing but it is useful for analysis and validation purposes.

As it can be inferred from the previous paragraphs, although correlation measurements are the basis of the interferometric MIRAS operation, the quality of the associated total power measurements, either in NIR or LICEF, are of utmost importance for providing the most reliable, stable, and accurate data.

\section{Power Measurement System (PMS)}

The PMS installed in all individual MIRAS receivers consist of a diode detector and associated circuitry to provide a dc 
voltage $v$ proportional to the total noise power

$$
v=G T_{\text {sys }}+v_{\text {off }}=G\left(T_{A}+T_{R}\right)+v_{\text {off }}
$$

where $G$ is the transducer power gain, $T_{\text {sys }}$ the system temperature, $T_{A}$ the antenna temperature, $v_{\text {off }}$ the instrumental offset, and $T_{R}$ the receiver equivalent noise temperature. Both gain and offset are periodically calibrated on board. The receiver noise temperature was measured on ground at different thermal conditions.

The system temperature that is needed to denormalize the digital correlations is directly solved from the aforementioned equation. The antenna temperature derived from this same equation is used only for the validation purposes [11].

\section{A. Gain}

In the current SMOS operations, PMS gains are calibrated every two months by internal hot/warm noise injection using seven sources and a distributed coaxial network that injects power to all LICEFs via input switches [9] [12]. The whole system is referred to as "calibration system" or CAS [3] [13]. This method relies on the knowledge of the differential noise power (hot minus warm) injected at each receiver's input. It is estimated by configuring the three NIRs to measure their input power when they are switched to an extra output port of the distribution network. A special mode called "NIR_R" is used for this purpose [14]. The PMS gain at the calibration input plane (CIP) for a particular receiver is then given by

$$
G^{C}=\frac{v_{H}-v_{W}}{\frac{\left|S_{L 0} f_{L}\right|^{2}}{\left|S_{N 0} f_{N}\right|^{2}}\left(T_{H}^{\mathrm{nir}}-T_{W}^{\mathrm{nir}}\right)}
$$

where $S$ refers to the distribution network S-parameters assuming the source at port 0 , the NIR at port $N$ and the LICEF at port $L$, and $T^{\text {nir }}$ is the NIR measurement in the NIR_R mode. Subscripts $H$ and $W$ refer to "hot" and "warm" levels, respectively. The parameters $f$ are the so-called "CAS factors" needed to correct small errors identified in the ground-measured $\mathrm{S}$ parameters. Three sets of CAS factors are used due to changes in operational modes: From launch to January 11, 2010, all arms operated in redundant mode; then arm "A" was switched to nominal; and in January 12, 2011, arm "B" was also switched to nominal mode. At each change, the CAS factors needed to be recomputed.

Equation (2) is only strictly applicable to LICEFs sharing a noise distribution network with a particular NIR. The complete algorithm for the actual distributed CAS design is detailed in [12]. In any case, this gain calibration method is ultimately based on the accurate NIR calibration and on the knowledge of the network S-parameters [15] after correction using the CAS factors.

To avoid introducing uncertainties due to NIR calibration stability (see Section III), a different approach has been independently implemented. As part of standard internal calibration procedures, all LICEF input ports are periodically switched to matched loads for about $17 \mathrm{~min}$ to inject uncorrelated noise (U-noise) needed for estimating the visibility offset [16]. This
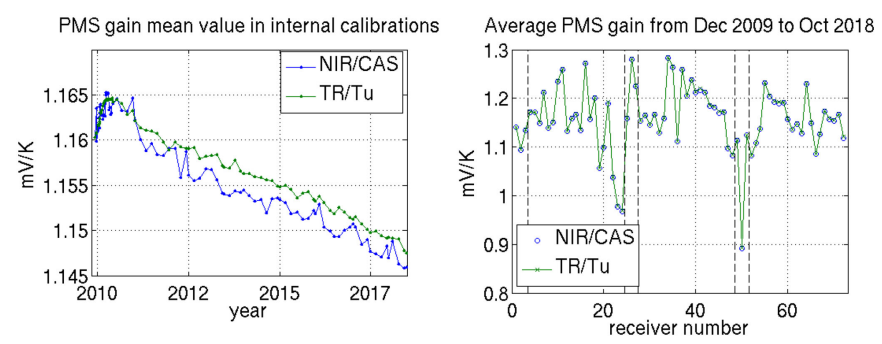

Fig. 1. Comparison of PMS gains calibrated by both methods: CAS/NIR and TR/Tu. Left: Average of all receivers versus time. Right: Time average for each receiver.

load includes a thermal sensor to monitor its temperature $T_{u}$. During this U-noise injection, the detected PMS output voltage (1) becomes $v_{u}=G^{C}\left(T_{u}+T_{R}^{C}\right)+v_{\text {off. }}$ The gain at the CIP is then readily solved as

$$
G^{C}=\frac{v_{u}-v_{\text {off }}}{T_{u}+T_{R}^{C}} .
$$

The receiver noise temperature at CIP is taken from the onground LICEF characterization as $T_{R}^{C}=T_{R}^{C}\left(T_{0}\right)+S_{\mathrm{TR}}\left(T_{u}-\right.$ $T_{0}$ ), where $T_{0}=21^{\circ} \mathrm{C}$ is the nominal characterization temperature and $S_{\mathrm{TR}}$ a sensitivity coefficient derived from measurements at different temperatures. Both $T_{R}^{C}\left(T_{0}\right)$ and $S_{\mathrm{TR}}$ are included in the MIRAS database. It could be argued that the receiver noise temperature may have changed in flight, resulting in introducing an error in the gain estimation (3). It has been observed, though, that its temporal stability is better than that of (2), which uses NIR on-orbit measurements. Fig. 1 (left) shows the average of all LICEF PMS gains along time using both approaches. After the first about six months (duration of the in-orbit commissioning phase or IOCP), in both cases, there is a constant negative trend, but in the case of NIR/CAS calibration, there is an increased ripple linked to the NIR instability. Consistency between both calibrations in all receivers is evidenced by the plot at the right of Fig. 1, where the average along time of the gain for all receivers is shown as a function of receiver number (see Table I). In all cases, both gain retrievals differ only by a small amount (about $0.1 \%$ difference).

This good consistency between the PMS gain absolute values is not as surprising as it may seem. The CAS factors are computed at three particular dates by forcing (1) and (3) to be equal. From January 12, 2011 (last CAS factors), both gains drift apart, as clearly seen in Fig. 1 .

\section{B. Offset}

The PMS offset is calibrated once a week using the four-point technique [17], a very robust method based on a two-level noise injection combined with an extra attenuation in the amplified signal just before the detection. The PMS offset depends only on the four corresponding voltages

$$
v_{\text {off }}=\frac{v_{H} v_{W_{L}}-v_{W} v_{H_{L}}}{\left(v_{H}-v_{H_{L}}\right)-\left(v_{W}-v_{W_{L}}\right)}
$$


TABLE I

CORRESPONDENCE BETWEEN RECEIVERS NUMBERING AND NAMES

\begin{tabular}{|cc|cc|c|c|}
\hline 1 & LCF_AB_03 & 25 & LCF_BC_03 & 49 & LCF_CA_03 \\
2 & NIR_AB_01_H & 26 & NIR_BC_01_H & 50 & NIR_CA_01_H \\
3 & NIR_AB_01_V & 27 & NIR_BC_01_V & 51 & NIR_CA_01_V \\
4 & LCF_A_01 & 28 & LCF_B_01 & 52 & LCF_C_01 \\
5 & LCF_A_02 & 29 & LCF_B_02 & 53 & LCF_C_02 \\
6 & LCF_A_03 & 30 & LCF_B_03 & 54 & LCF_C_03 \\
7 & LCF_A_04 & 31 & LCF_B_04 & 55 & LCF_C_04 \\
8 & LCF_A_05 & 32 & LCF_B_05 & 56 & LCF_C_05 \\
9 & LCF_A_06 & 33 & LCF_B_06 & 57 & LCF_C_06 \\
10 & LCF_A_07 & 34 & LCF_B_07 & 58 & LCF_C_07 \\
11 & LCF_A_08 & 35 & LCF_B_08 & 59 & LCF_C_08 \\
12 & LCF_A_09 & 36 & LCF_B_09 & 60 & LCF_C_09 \\
13 & LCF_A_10 & 37 & LCF_B_10 & 61 & LCF_C_10 \\
14 & LCF_A_11 & 38 & LCF_B_11 & 62 & LCF_C_11 \\
15 & LCF_A_12 & 39 & LCF_B_12 & 63 & LCF_C_12 \\
16 & LCF_A_13 & 40 & LCF_B_13 & 64 & LCF_C_13 \\
17 & LCF_A_14 & 41 & LCF_B_14 & 65 & LCF_C_14 \\
18 & LCF_A_15 & 42 & LCF_B_15 & 66 & LCF_C_15 \\
19 & LCF_A_16 & 43 & LCF_B_16 & 67 & LCF_C_16 \\
20 & LCF_A_17 & 44 & LCF_B_17 & 68 & LCF_C_17 \\
21 & LCF_A_18 & 45 & LCF_B_18 & 69 & LCF_C_18 \\
22 & LCF_A_19 & 46 & LCF_B_19 & 70 & LCF_CC19 \\
23 & LCF_A_20 & 47 & LCF_B_20 & 71 & LCF_C_20 \\
24 & LCF_A_21 & 48 & LCF_B_21 & 72 & LCF_C_21 \\
\hline
\end{tabular}
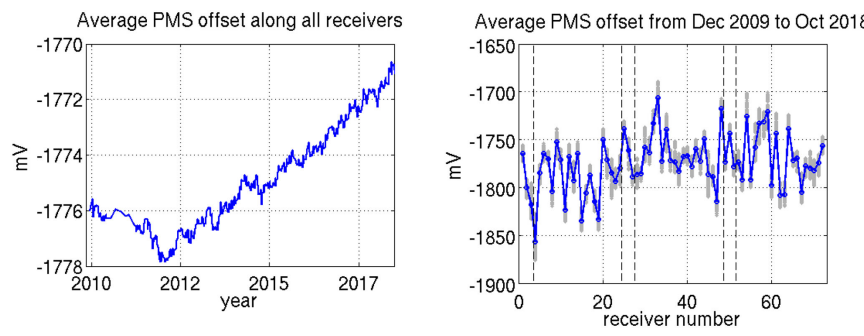

Fig. 2. PMS instrumental offset. Left: Average of all receivers versus time. Right: Time average for each receiver. The gray points are all the individual values for each receiver.

where the subscript $L$ indicates that the attenuator is ON. Fig. 2 shows all PMS offset retrievals averaged along receivers (at left) and along time (at right). After an initial decrease, the average offset is increasing steadily since 2012. The range of offset values for each receiver is kept inside reasonable limits with respect to its mean.

In general, the offset is very stable, with a sensitivity coefficient with respect to the temperature of the order of $0.2 \mathrm{mV} / \mathrm{K}$. However, two artifacts limit in practice its quality: erratic voltage jumps and heater signal interference. The first one is analyzed in the next paragraphs and the second one in Section II-D.

While routinely analyzing calibration parameters, it was eventually observed after the IOCP that the PMS offset voltages experienced erratic abrupt jumps. To lower their impact on data quality, on March 2011, it was decided to reduce the PMS offset intercalibration period from eight weeks to one. As an illustration, Fig. 3 (left) shows the PMS offset evolution along the mission of four receivers chosen at random. Sharp jumps of several millivolts are clearly seen in all of them. Note the small number of measurements between July 2010 (end of IOCP) and March 2011.
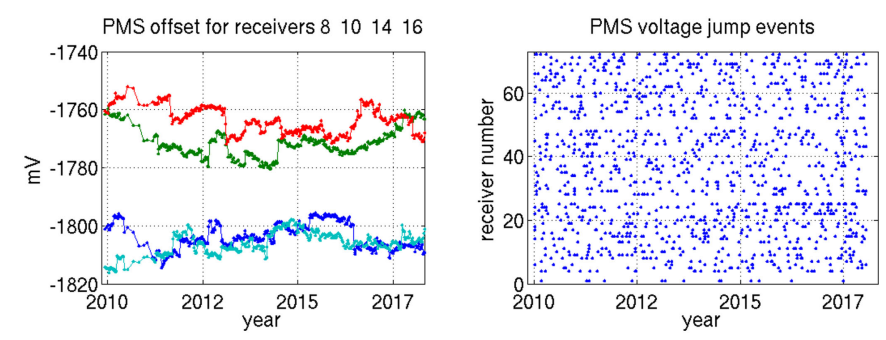

Fig. 3. Left: PMS offset of randomly selected receivers to illustrate the voltage jumps. Right: Detected voltage jumps for all receivers along the mission.

In order to better estimate the precise time of a jump, systematic PMS voltages of daily ascending and descending orbits over a limited zone on the Pacific ocean have been analyzed. To increase the consistency among different receivers, PMS voltages are first converted to antenna temperatures solving the second part of (1) and using time-constant calibration parameters (gain, offset, and noise temperature). For each receiver, the difference $\left(\Delta T_{A}\right)$ between its antenna temperature and the median of antenna temperatures of all receivers is computed. Retrievals of $\Delta T_{A}$ are averaged over a relatively large region, ending with one single value every orbit, or equivalently, $12 \mathrm{~h}$. Then, temporal series of averaged $\Delta T_{A}$ are analyzed and a jump is detected if the difference between two consecutive values is larger than a threshold established as $2.8 \mathrm{~K}$. This value has been adopted after a careful tradeoff that maximizes jump detection while minimizing the number of false alarms due to noise and/or ascending versus descending differences. Moreover, jumps are only considered if they happen simultaneously in both polarizations. The jump event time is estimated just in the middle of the two times between which a jump has been detected, which means $6 \mathrm{~h}$ after the first point. The result is that jumps are produced quite randomly at an average rate of two to three jumps per year per receiver. Fig. 3 at right shows the jump times for each receiver along the mission. NIR receivers (numbers 2, 3, 26, 27, 50, and 51 according to Table I) are discarded from this analysis since their PMS voltages during science measurements are invalid. Due to the dynamic noise injection in the NIR operation [14], the total power at input, and hence, the PMS voltage, remains constant independently of the scene.

In nominal operation, a PMS voltage jump is automatically corrected by the next offset calibration event. To reduce the time window in which a given jump is not yet canceled, it is proposed to start applying the next PMS offset calibration at the estimated jump time. As an example, Fig. 4 shows the antenna temperature of receiver LCF_A_12 (see Table I) before and after applying the correction. Jumps and offset calibration events are shown as vertical lines to visualize that, when the proposed procedure is applied, the correction is effective at the jump time, with an uncertainty of only $\pm 6 \mathrm{~h}$, instead of the next offset calibration event, sometimes, a couple of days afterwards.

This correction mechanism can only be applied to reprocessing campaigns since a previous processed data are required to determine the exact offset jump times. 


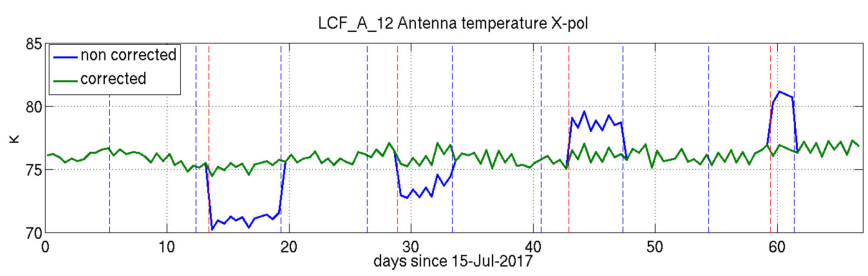

Fig. 4. PMS voltage jumps correction. Blue dashed lines indicate offset calibration events. Red dashed lines show estimated jump times.

\section{Linearity Correction}

Preliminary investigations on the PMS linearity [18] showed that its behavior is better modeled by means of a second-order response $v=a T_{\text {sys }}^{2}+G T_{\text {sys }}+v_{\text {off }}$ instead of the linear model (1). The impact of neglecting the second-order term was estimated in [18] to be of the order of $0.4 \%$, which is not completely negligible.

The PMS flight units nonlinear behavior was characterized on the ground by the deflection method [19]. Each unit was driven by a noise source followed by a stepped attenuator producing 11 different input noise power levels. At each one, extra noise power was injected during a short time to measure the slope of the detector characteristic input-output curve. For a given input level $i$, the deflection ratio is defined as $D_{i}=\Delta v_{i} / \Delta v_{1}$, where $\Delta v$ is the voltage increment when the extra noise is injected. For an ideal linear detector, this parameter should be unity for all input levels, but in real detectors, it departs from unity at high input powers. Using this approach, nonlinearities are observed as deviations of the noise diode deflection as the input noise temperature changes. The detector is then characterized by the empirical model proposed in [20]: the equivalent linear voltage is related to the actual detected voltage by

$$
v_{\text {lin }}=C \sqrt{1+\frac{2 v_{\mathrm{det}}}{C}}-C
$$

where $C$ is a characterization constant with units of $V$. At each input power level, the deflection ratio $D$ was measured and the parameter $C$ computed for all receivers by a minimization algorithm [19].

Equation (5) applies only to voltages without instrumental offset, so this one must first be subtracted from the raw detected voltage. The procedure starts by computing a first guess of the offset using the measured voltages in (4). The result is then subtracted to the voltages and (5) is applied to correct for nonlinearity. Not surprisingly, this linearized voltage has nonzero offset when computed again using (4). This "residual" offset is then added to the initial guess.

Fig. 5 is a simulation intended to justify this procedure. A second-order polynomial relating voltage to the system temperature is considered with typical PMS gain (first-order term) and offset (zero-order term) but with an exaggerated tenfold secondorder term. While the original offset is set to $-1766.51 \mathrm{mV}$, the estimated value using (4) results in $-1704.38 \mathrm{mV}$. When this is subtracted from the original voltages, the blue line of the plot at
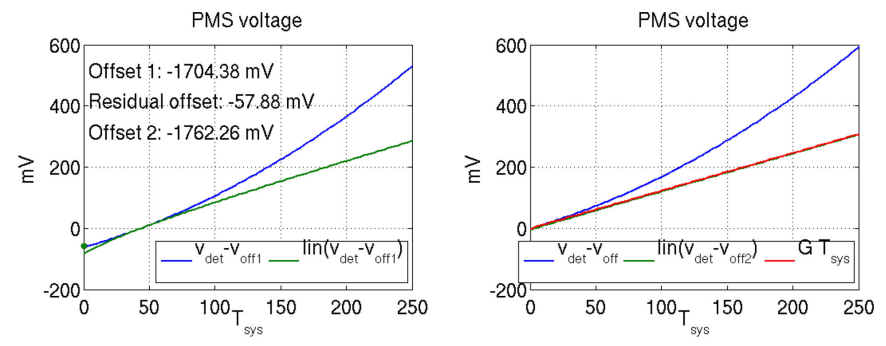

Fig. 5. Example of the linearity correction with simulated data. Left: First offset substraction and linearization. Right: Original and linearized voltages compared to an ideal linear system.

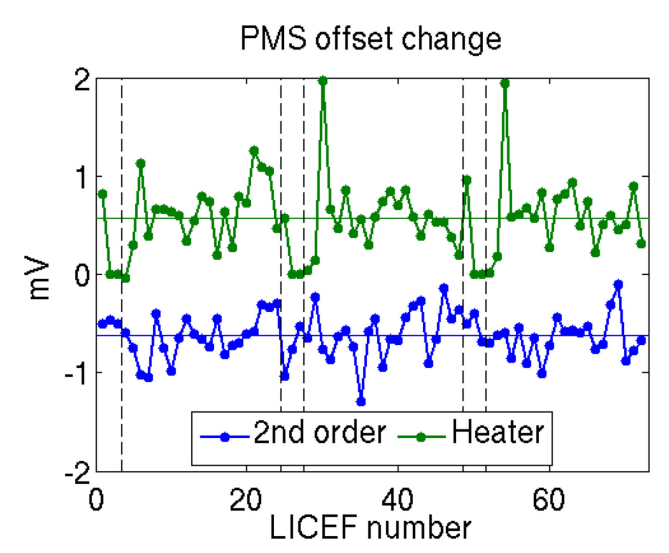

Fig. 6. Impact of second-order and heater corrections in PMS offset.

the left of Fig. 5 is obtained. Linearization using (5) converts it to the green line of the same figure. Equation (4) applied on this result produces a residual offset of $-57.88 \mathrm{mV}$ that is added to the first value to give a final offset of $-1762.26 \mathrm{mV}$, only 4-mV apart from the original. The plot at the right shows in blue the original voltages without offset, and in green, the linearized voltage after subtracting the final estimated offset. For comparison, the red line (practically overlapped to the green one) shows a linear model with no offset. The linearization approach does reduce significantly the system temperature estimation error. For the actual detectors, much more linear than this simulation, the results are even better.

Due to this adjustment, the second-order correction introduces a constant negative difference of about $0.6 \mathrm{mV}$ in the offset with respect to not correcting the linearity. Fig. 6 (blue line) shows the difference in the PMS offsets between applying or not the second-order correction. This difference is constant in time so it only affects the absolute calibration, equivalently to about $0.6 \mathrm{~K}$ in the system temperature, and not the temporal stability.

\section{Heater Correction}

MIRAS has a temperature stabilization system that tries to keep the temperature within a relatively narrow operational margin. Due to the large dimensions of the instrument, the temperature control is distributed along different parts. Each control and 
monitoring node $(\mathrm{CMN})$ has its own thermal sensor that drives a heater ON/OFF to compensate for thermal swings. The result is that $\mathrm{CMN}$ temperatures are not perfectly constant but have a cyclic behavior around a mean value.

A clear dependence of the PMS voltages on the heater status was identified during the IOCP [21]. There are receivers almost insensitive, but for others, it is very evident. This phenomenon is generated in the CMN dc amplifiers (after detection) and is due to the thermal excursions of this circuit produced by the heaters. It is not linked to the front-end temperature, for which, a different sensitivity coefficient is independently derived. In [21], a correction based on a delayed version of the heater signal was proposed and it was implemented in the level 1 operational processor up to version 620. However, careful analysis of LICEF antenna temperatures over the ocean showed that this solution was overcorrecting. A new strategy based on a double exponential model with empirical coefficients applied to the absolute instantaneous voltages was then proposed. This approach efficiently corrects heater dependence and has been implemented in the new version of the processor (L1OP v710).

Conceptually, an offset that depends on the voltage level is a gain, so this new solution suggests that both PMS gain and offset depend on the heater status. A model for both using exponential functions derived from the heater control signal is here proposed. To confirm this hypothesis, data acquired during the "electrical stability test" of April 20 and 22, 2010 and the so-called "E3" test of February 9, 2010 have been reprocessed. The first one consisted of continuous internal calibration sequences commanded during almost two days. It has been used for the gain. The second one consisted of continuous four-point sequences during $10 \mathrm{~h}$ to get only the offset. Fig. 7 shows both gain and offset as they are measured from the corresponding calibration sequences, together with the corresponding heater signal and the exponential model. The delay model for the offset [21] is also shown in the plot at bottom. As it is seen, both gain and offset are accurately modeled by exponential functions for both rise and decay.

Both gain and offset depend also on the front-end physical temperature, characterized on ground by a sensitivity parameter [9], [22]. The full linear model for the PMS voltage is then

$$
v=\left[G_{0}+S_{G} \Delta T+f_{G}(H)\right] T_{\text {sys }}+\left[v_{\text {off }}+S_{\text {off }} \Delta T+f_{\text {off }}(H)\right]
$$

where $f_{G}(H)$ and $f_{\text {off }}(H)$ are, respectively, the models of the gain and offset as a function of the heater control signal $(H)$ shown in Fig. 7. Note that these two models are opposite in sign with respect to the heater signal, and depending on the $T_{\text {sys }}$ value, they may even cancel each other in (6). This is the reason why correcting only the offset failed.

Heater correction consists of subtracting the models $f_{G}(H)$ and $f_{\text {off }}(H)$ to the gain and offsets in order to recover constant values for them. As an example, Fig. 8 shows individual retrievals of the PMS gain and offset for a particular receiver; and the corresponding corrected values using either only temperature or also the heater signal. Applying only temperature correction does not stabilize the gains nor the offsets, so the need of the heater correction is evident. For completeness, the
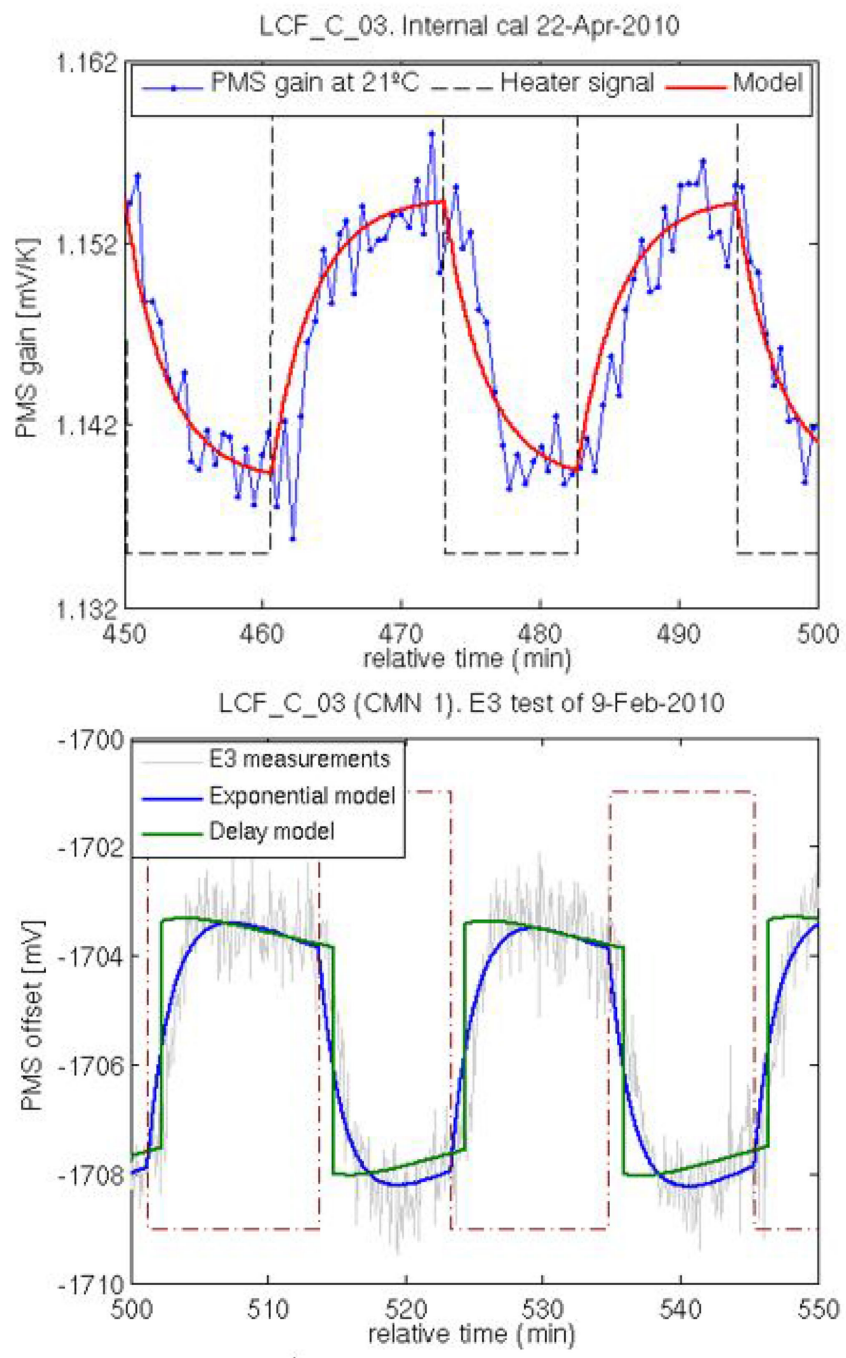

Fig. 7. Heater dependence and exponential model. Top: Gain. Bottom: Offset. In this case, the delay model is also shown.

figure also shows the front-end physical temperature and the heater signal.

This dual gain/offset correction has demonstrated to effectively cancel heater dependence in antenna temperature for all cases. For example, Fig. 9 shows the LICEF antenna temperature computed by solving the second part of (1) in three different cases: with no heater correction, correcting only the offset and correcting both offset and gain. Front-end temperature dependence is always corrected. Only the last case is able to cancel the heater dependence. For comparison, the antenna temperature measured by the NIR (see Section III) is included in the figure. Also included is the front-end physical temperature. The plot at the top corresponds to an ocean target, while the one at bottom is for cold sky, in the middle of an external calibration maneuver.

It is interesting to observe that, for the ocean case, the result with no correction is well behaved and clearly better than correcting only the offset. This is so because gain and offset heater dependencies compensate each other. On the other hand, for the 

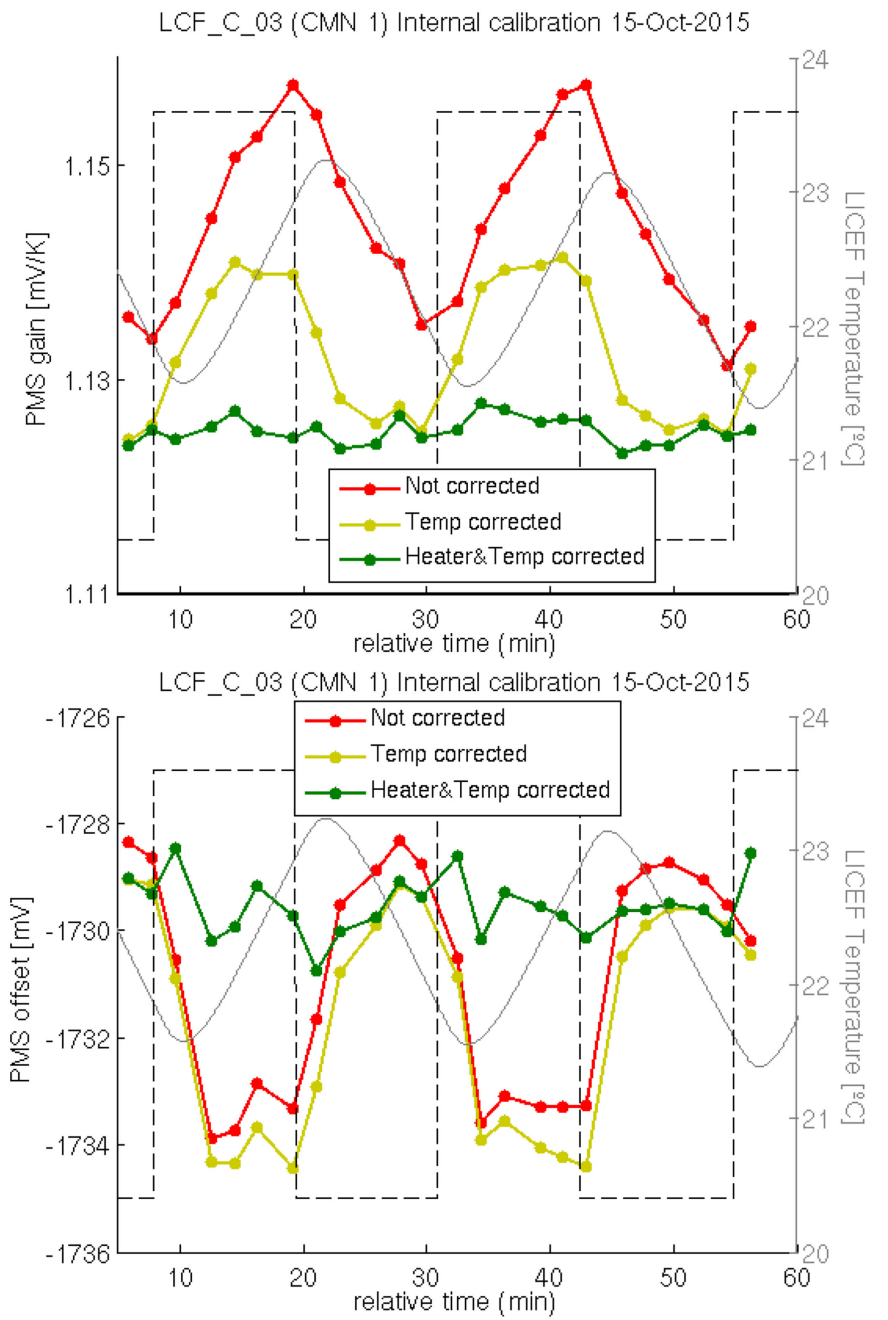

Fig. 8. Effect of correcting PMS calibration parameters with respect to frontend temperature and heater signal. Top: Gain, Bottom: offset. Gray solid line is the physical temperature and dashed gray line the heater signal.

sky antenna temperature, the effect of the heater is clearly seen in the noncorrected case. Applying both gain and offset corrections is effective, although not perfect, but applied only to the offset clearly overcorrects.

\section{E. Front-End Loss}

Since the PMS gain is internally calibrated, the total frontend loss between antenna and calibration planes must be taken into account. The contribution of the input switch itself was measured on-ground using a network analyzer while the antenna ohmic loss was initially estimated as $0.3 \mathrm{~dB}$. However, these default values are not fully consistent with the observations [23].

In-flight characterization of the total front-end loss is carried out using external maneuvers performed every two weeks. While pointing to the cold sky, the receivers' input switch is sequentially commuted to a matched load and to the antenna. With these two measurements, assuming that the front-end physical
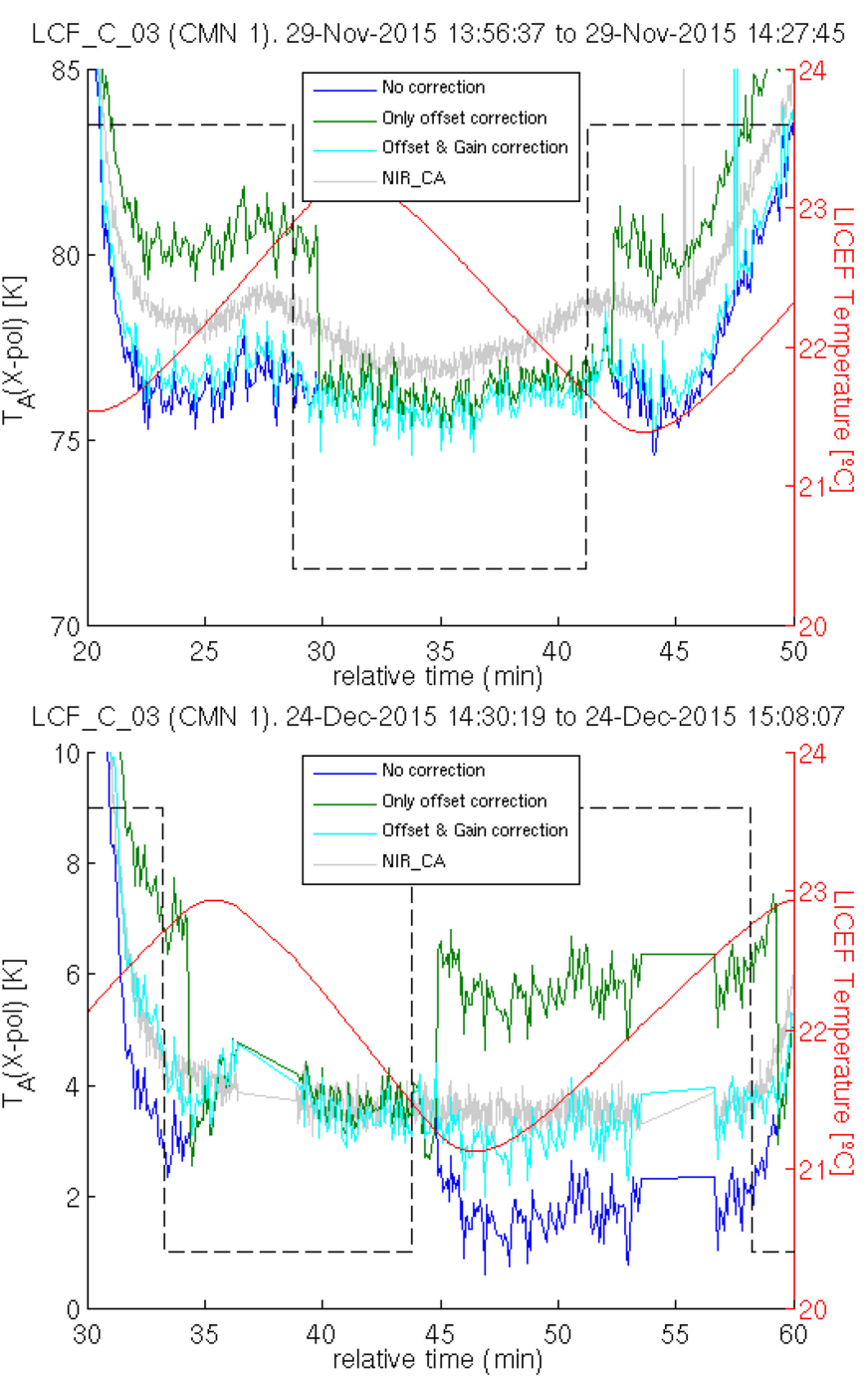

Fig. 9. LICEF antenna temperature showing the heater dependence for both ocean (top) and sky (bottom) views. The "only offset correction" consists of a constant correction depending on a delayed heater signal. The heater signal is shown in dashed lines.

temperature is homogeneous and equal to that of the matched load, the gain and receiver noise temperature at the antenna plane can be estimated by the standard two-level calibration equations

$$
G=\frac{v_{\text {sky }}-v_{u}}{T_{\text {sky }}-T_{u}} \quad T_{R}=\frac{v_{u}^{\prime} T_{\text {sky }}-v_{\text {sky }}^{\prime} T_{u}}{v_{\text {sky }}-v_{u}}
$$

where $v^{\prime}=v-v_{\text {off }}$ and the offset is calibrated independently during the same maneuver. The subscript $u$ refers to the matched load and the subscript sky to the external target. The antenna temperature of the sky is considered as a calibration standard and is computed as

$$
T_{\text {sky }}=\frac{D}{4 \pi} \iint_{4 \pi} T_{B \text { sky }}(\Omega)\left|F_{n}(\Omega)\right|^{2} d \Omega
$$

where $T_{B \text { sky }}$ is the brightness temperature of the sky including the Galaxy, the cosmic background and other sources [24], $\left|F_{n}\right|^{2}$ 

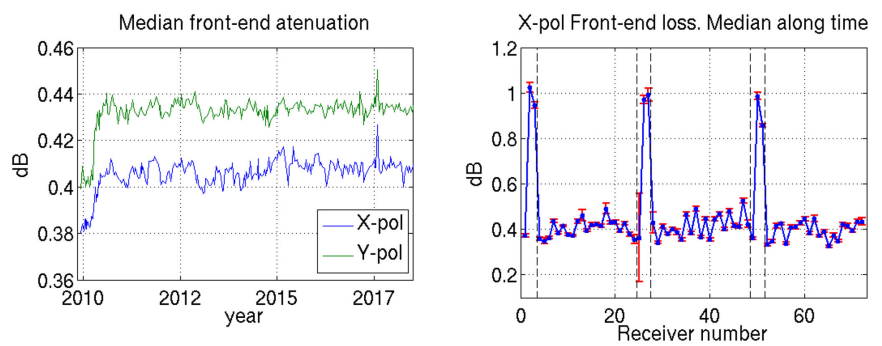

Fig. 10. LICEF front-end loss estimated from external calibrations. Left: Median of all LICEF as a function of time. Right: Temporal median for each LICEF or NIR receiver. The error bars show one-sigma spread.
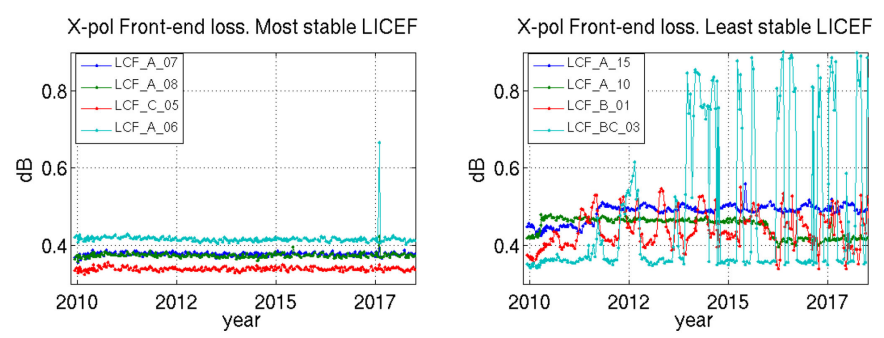

Fig. 11. Evolution of some LICEF front-end loss. Left: Four most stable receivers. Right: Four least stable receivers (excluding NIR).

is the normalized antenna power pattern measured on ground, $D$ is the antenna directivity, and $\Omega$ the solid angle. Antenna back lobes are neglected in this computation.

On the other hand, the PMS gain at the internal calibration plane can be computed with (3) using the voltage measured with the switch at $u$ position. The total front-end loss is then estimated as the ratio of internal to external gains

$$
L_{\mathrm{FE}}=\frac{G^{C}}{G}
$$

Fig. 10 shows the results: In the left plot, there is the median of all LICEF (excluding NIR) front-end loss as a function of time. A systematic increase is seen during the IOCP (up to mean July 2010), but then, the results stabilize. Some ripples are still seen, especially in X-polarization, until 2014. The spike in August 2017 is present in almost all receivers so it is probably due to the presence of RFI. The plot at right shows the temporal median for each LICEF or NIR receiver adding error bars at one standard deviation. Only X-polarization antennas are shown for simplicity, but similar results are found for Y-polarization. NIR front ends (receivers 2, 3, 26, 27, 50, and 51) have larger losses (about $1 \mathrm{~dB}$ ) due to the coupler and cables used to inject noise just after the antenna (see Fig. 12). In general, this plot shows that antenna loss is very stable for most receivers except for one in particular (number 25) that shows a very large variation. Fig. 11 shows the front-end loss evolution of the four most stable LICEF (left) and the four most unstable ones (right) excluding NIRs (they are given in Fig. 15). Most of the receivers behave as in the left plot, with front-end loss essentially constant along time. But there are some of them, as for example, those shown in the plot at right, that have seasonal variations (LCF_B_01) or abrupt

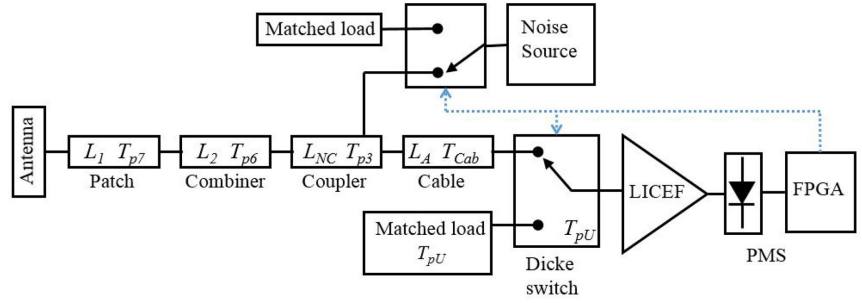

Fig. 12. NIR block diagram. The different attenuators were characterized on ground and physical temperatures are measured on flight.

jumps (LCF_A_10) or large spikes (LCF_BC_03). All these artifacts are probably originated at the antenna to the front-end connection or at the input switch. For operational processing, constant front-end loss values are used. They were computed once as the average of the corresponding retrievals obtained in four external maneuvers (15-Oct-2014, 29-Oct-2014, and 26Nov-2014).

\section{NOISE INJECTION RADIOMETERS (NIRS)}

Three NIRs are installed near the center of the MIRAS array with the main purpose of measuring the mean antenna temperature, or zero-spacing visibility [14], [25]. Each NIR is formed by two receiver chains (LICEFs) connected to a single dual polarization antenna and additional circuitry to perform the noise injection operation. Fig. 12 shows the block diagram for one polarization. The antenna loss is split into two elements, one corresponding to the radiation patch, at outside temperature $T_{p 7}$, and the other to the microstrip polarization combiner, at internal temperature $T_{p 6}$. The antenna output is connected to a directional coupler at temperature $T_{p 3}$ to allow injecting power from the internal noise source. A coaxial cable, at temperature $T_{\text {Cab }}$, connects the coupler output to the Dicke switch at temperature $T_{p U}$, already inside the LICEF unit. The matched load at the other port of the Dicke switch is also at temperature $T_{p U}$ and is actually integrated in the switch. The LICEF PMS output is sent to an FPGA with some logic to drive both switches. The Dicke switch commutes periodically between the matched load and the antenna path at a rate of $26.6 \mathrm{hz}$ with a $50 \%$ duty cycle. Consequently, during half of the Dicke cycle, the noise injected to the LICEF input has temperature $T_{p U}$, equivalent to injecting at antenna plane noise power with temperature given by

$$
\begin{aligned}
T_{U}^{A}= & L_{1} L_{2} L_{\mathrm{NC}} L_{A} T_{p U}-T_{p 7}\left(L_{1}-1\right) \\
& -L_{1} T_{p 6}\left(L_{2}-1\right)-L_{1} L_{2} T_{p 3}\left(L_{\mathrm{NC}}-1\right) \\
& -L_{1} L_{2} L_{\mathrm{NC}} T_{\mathrm{Cab}}\left(L_{A}-1\right)
\end{aligned}
$$

where all the attenuations and temperatures are defined in Fig. 12. If all NIR components were kept at the same physical temperature $T_{p U}$, then this equation would be reduced to $T_{U}^{A}=T_{p U}$.

During the other half of the cycle, the Dicke switch is connected to the antenna path. Simultaneously, for a fraction $\eta$ of the half cycle, noise from the internal source is injected through 
TABLE II

NOMINAL ATTENUATION OF NIR FRONT-END COMPONENTS

\begin{tabular}{|c|r|r|r|r|}
\hline NIR & $L_{1}[\mathrm{~dB}]$ & $L_{2}[\mathrm{~dB}]$ & $L_{N C}[\mathrm{~dB}]$ & $L_{a}[\mathrm{~dB}]$ \\
\hline NIR_AB_H & 0.05 & 0.11 & 0.316 & 0.164 \\
NIR_AB_V & 0.05 & 0.07 & 0.220 & 0.158 \\
NIR_BC_H & 0.05 & 0.12 & 0.197 & 0.163 \\
NIR_BC_V & 0.05 & 0.15 & 0.219 & 0.161 \\
NIR_CA_H & 0.05 & 0.07 & 0.208 & 0.156 \\
NIR_CA_V & 0.05 & 0.12 & 0.194 & 0.157 \\
\hline
\end{tabular}

the coupler. The equivalent noise temperature at antenna plane during this half cycle becomes

$$
T_{a}^{A}=\eta\left(T_{A}+T_{\mathrm{NA}}\right)+(1-\eta) T_{A}
$$

where $T_{A}$ is the antenna temperature and $T_{\mathrm{NA}}$ a parameter related to the source excess noise ratio. It can be viewed as the equivalent noise temperature injected at antenna plane. The logic inside the FPGA drives the switches so that always the equivalent temperatures in both halves of the Dicke cycle are equal, or $T_{U}^{A}=T_{a}^{A}$. In consequence, the antenna temperature can be solved as

$$
T_{A}=-\eta T_{\mathrm{NA}}+T_{U}^{A} .
$$

This is a standard radiometer equation in which the raw measurement is the fraction of half Dicke cycle $\eta$, the gain is $-T_{\mathrm{NA}}$ and the offset is $T_{U}^{A}$. This one is computed at each measurement epoch using (10), so there is only one unknown to be determined by calibration, $T_{\mathrm{NA}}$. It is operationally measured every two weeks during external maneuvers by solving (12) and assuming that the antenna temperature is equal to $T_{\text {sky }}$, given by (8).

The physical temperatures needed in (10) are measured by thermal sensors installed in the different NIR components (see Fig. 12). The corresponding attenuations are fixed values characterized on ground and provided in Table II. All components except the antenna patch are enclosed in a thermally controlled environment, so all temperatures except $T_{p 7}$ are very similar and quite constant. This fact together with the low attenuation values shown in Table II means that $T_{U}^{A}$ in (10) becomes very similar to the physical temperature $T_{p U}$. Fig. 13 shows the different equivalent temperatures of one particular NIR for a complete orbit that includes a sky look. As predicted, $T_{U}^{A}$ is very similar to $T_{p U}$ although there is a small but visible effect of the impact of $T_{p 7}$ when this one drops down due to the rotation of the instrument to point to the cold sky. Not surprisingly, physical temperatures $T_{p 3}$ and $T_{p 6}$ are almost identical. The outside temperature $T_{p 7}$ is always lower than all the others and drops drastically when the instrument rotates to point the cold sky.

\section{A. Front-End Loss On-Flight Characterization}

During the IOCP, it was suspected that the NIR front-end attenuator values of Table II needed to be refined. The most critical one is that of the antenna patch $L_{1}$, since in (10), it is multiplied by the external temperature $T_{p 7}$, highly varying and not subject to thermal control. Any inaccuracy in this attenuator would produce artifacts on the science products linked to the external

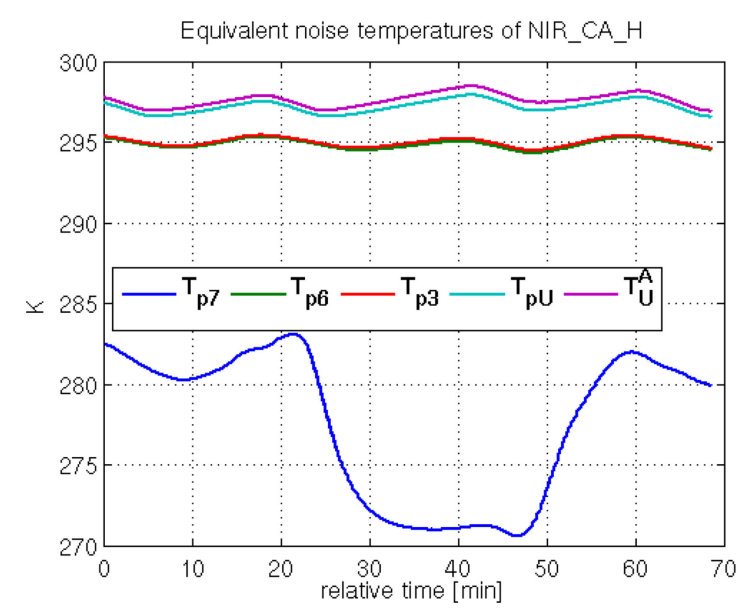

Fig. 13. Equivalent noise temperature for NIR during an orbit. The drop in $\mathrm{Tp} 7$ is due to an external maneuver.

temperature. These artifacts were indeed observed on the data initially processed, and for the second mission, reprocessing $L_{1}$ was empirically changed to $0.15 \mathrm{~dB}$ instead of the $0.05 \mathrm{~dB}$ of Table II. The data stability improved significantly but still some residual variations persist, especially in X-polarization.

A new approach to estimate the $L_{1}$ attenuation is proposed. First, for a given dataset, a zeroth-order antenna temperature is computed by approximating $T_{U}^{A} \approx T_{p U}$ in (12), resulting in $\tilde{T}_{A}=-\eta T_{\mathrm{NA}}+T_{p U}$. This equation does not use any supposedly known value of $T_{p 7}$, so any dependence found with respect to this temperature must be solely attributed to be intrinsic to the instrument. Note also that $\tilde{T}_{A}$ is also independent of the assumed values of the different attenuations $\left(L_{1}, L_{2}, L_{\mathrm{NC}}\right.$, and $L_{a}$ ). On the other hand, since all temperatures other than $T_{p 7}$ are similar (see Fig. 13), the actual antenna temperature can be reasonably well estimated using a two-temperature model with only $T_{p 7}$ and $T_{p U}$, that is considering $T_{p 3} \approx T_{p 6} \approx T_{p U}$ in (10). In this case, the antenna temperature (12) becomes $T_{A}=-\eta T_{\mathrm{NA}}+T_{p U} L_{1}-T_{p 7}\left(L_{1}-1\right)$. This result can be expressed as a function of the zeroth-order antenna temperature as

$$
\tilde{T}_{A}=T_{A}+\left(L_{1}-1\right)\left(T_{p 7}-T_{p U}\right)
$$

and $L_{1}$ can be retrieved from the slope of $\tilde{T}_{A}$ with respect to $\left(T_{p 7}-T_{p U}\right)$.

In order to use this equation accurately, all (or most) dependencies of $T_{A}$ with other parameters should be first canceled. The method has then been implemented using the difference between ascending and descending orbits, separated by only $12 \mathrm{~h}$, passing over the same area. Since the Sun hits the instrument differently when ascending with respect to descending, the external temperature $T_{p 7}$ changes accordingly. All parameters related to the surface emission are expected to be very similar. To avoid antenna temperature contributions from the sky, the reconstructed brightness temperature averaged in the alias-free field of view is used instead of the antenna temperature. In this situation, the only possible sources of change, besides $T_{p 7}$, are 

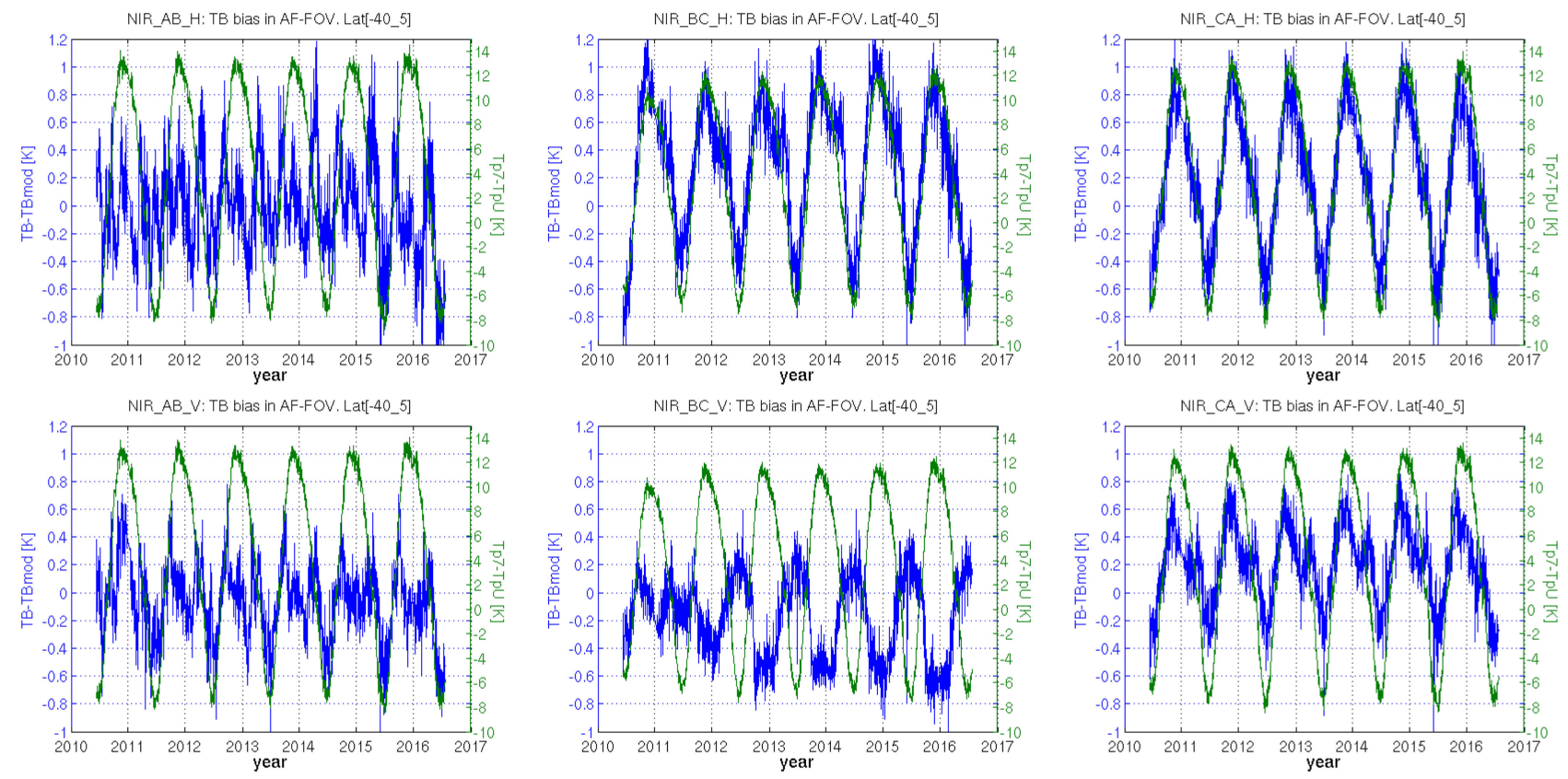

Fig. 14. Descending minus ascending difference of the brightness temperature bias with respect to a model and corresponding difference in physical temperatures.

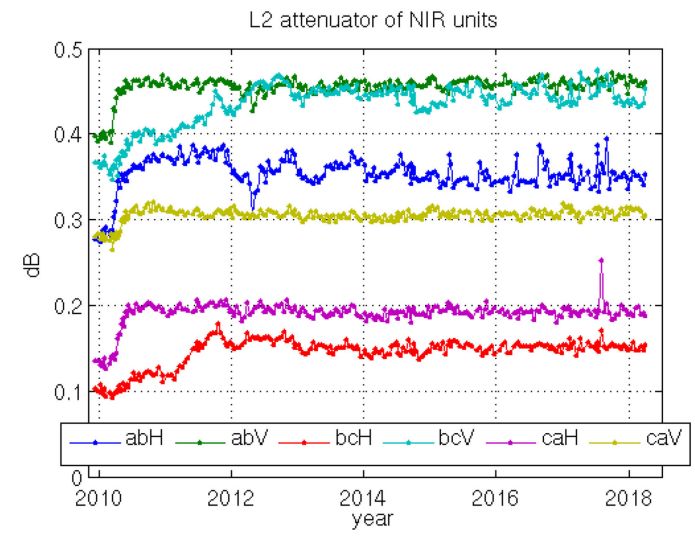

Fig. 15. NIR $L_{2}$ attenuator values derived from PMS gain ratio in external maneuvers (see Section II-E).

the faraday rotation angle and the galactic glint, both different between ascending and descending orbits. They have been considered by subtracting the reconstructed brightness temperature from a model that includes these effects. To have stable data and model, only orbits passing over the Pacific ocean have been considered, one ascending and one descending per day from mid 2010 to mid 2016, totaling 3800. All data in the latitude range from $-40^{\circ}$ to $5^{\circ}$ are averaged to get a single measurement point.

Results are shown in Fig. 14 where plots of brightness temperature bias with respect to the model are provided superimposed to plots of the difference $T_{p 7}-T_{p U}$. Units NIR_BC_H and NIR_CA_H behave as expected, showing a very good correlation between both parameters. NIR_CA_V shows some deviations in the positive cycles, NIR_AB_H and NIR_AB_V do not seem to depend on $T_{p 7}$ and NIR_BC_V even shows a negative correlation, which is highly unexpected. One possible reason for these discrepancies may be a dependence of the attenuator itself $L_{1}$ with the temperature, for example, due to mismatch, that could compensate the variation due to the noise emitted by $L_{1}$. However, all attempts to introduce this dependence have been unsuccessful. It might also be that the units with less dependence have effectively lower attenuation, but this is somewhat contradictory with the fact that the most stable unit is precisely NIR_CA, for which the dependence is more clear (see Fig. 16).

Linear regression between blue and green plots of Fig. 14 provides the slopes, from which, subtracting 1 , the corresponding $L_{1}$ is obtained according to (13). Table III gives the results for all NIR units, indicating also the correlation coefficient. For the two units with a higher correlation coefficient (NIR_BC_H and NIR_CA_H), $L_{1}$ is of the order of $0.3 \mathrm{~dB}$, significantly larger than the original prediction provided in Table II. This fact could indicate that the effective attenuation affected by the external temperature is not restricted to the microstrip patch antenna, for which loss is expected to be much lower, but it includes at least part of the microstrip polarization combiner. This is consistent with the fact that different behavior is observed, for a given NIR unit, in each polarization, although the radiating path and the temperature $T_{p 7}$ are both the same.

On the other hand, NIR_AB in both polarizations has low correlation coefficient and the computed attenuation is more consistent with the original values of Table II. The negative correlation of NIR_BC_V is unexpected, so $L_{1}$ is set to zero decibel for this unit.

Once $L_{1}$ is characterized, $L_{2}$ can be estimated as $L_{2}=$ $L_{\mathrm{FE}} /\left(L_{1} L_{\mathrm{NC}} L_{A} L_{D A}\right)$ (see Fig. 12) where $L_{\mathrm{FE}}$ is the total 

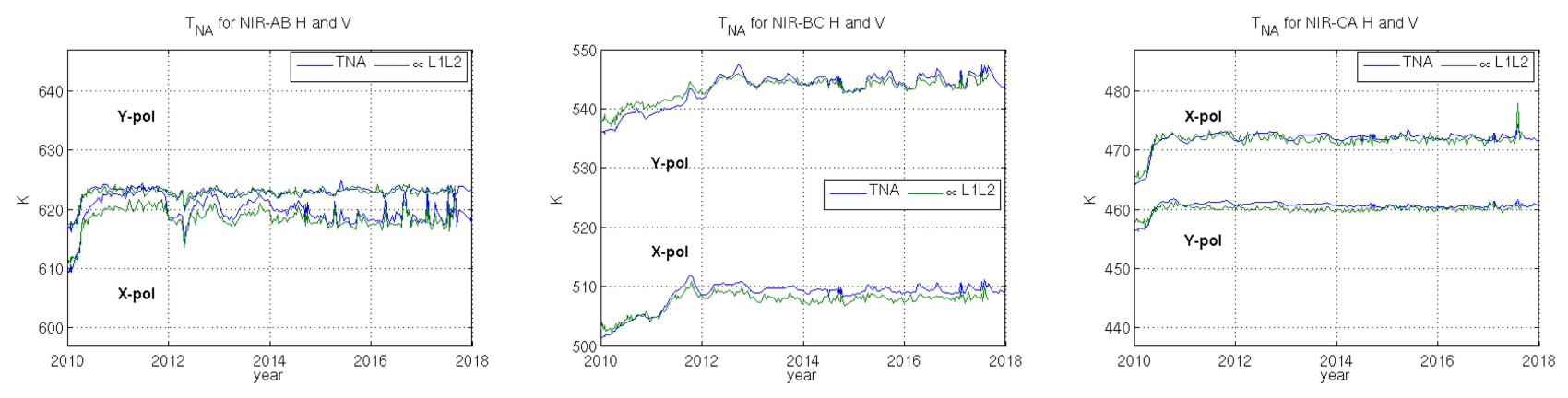

Fig. 16. Relation between front end loss and $T_{\mathrm{NA}}$.

TABLE III

OPTIMIZED $L_{1}$ ATTENUATION AND CORRELATION COEFFICIENT $R$ OF THE PLOTS OF FIG. 14

\begin{tabular}{|c|r|r|r|}
\hline NIR & $L_{1}[\mathrm{~dB}]$ & \multicolumn{1}{|c|}{$\mathrm{R}$} & $L_{2}[\mathrm{~dB}]$ \\
\hline NIR_AB_H & 0.029 & 0.136 & 0.32 \\
NIR_AB_V & 0.081 & 0.462 & 0.37 \\
NIR_BC_H & 0.289 & 0.892 & 0.14 \\
NIR_BC_V & -0.157 & -0.721 & 0.43 \\
NIR_CA_H & 0.268 & 0.937 & 0.19 \\
NIR_CA_V & 0.141 & 0.777 & 0.30 \\
\hline
\end{tabular}

Averaged values of $L_{2}$ are given in last column.

front-end loss obtained from the external to internal PMS gain ratio (9) in Section II-E. Results provided in Fig. 15 show that the most stable units in terms of the front-end loss are NIR_CA_H, NIR_CA_V, and NIR_AB_V, although all have a transient at the beginning of the mission until mid 2010. This effect is common to all antennas and is also observed in the average front-end attenuation of Fig. 10. The spike in August 2017 was already commented in relation to Fig. 10. Average values of $L_{2}$ have been derived from these data using the same selected maneuvers considered for the LICEF front-end loss (see Section II-E). They are provided in the last column of Table III.

\section{B. NIR External Calibration}

The NIR gain $T_{\mathrm{NA}}$ is computed at each external maneuver by solving (12) and assuming $T_{A}=T_{\text {sky }}(8)$, so $T_{\mathrm{NA}}=$ $\left(T_{U}^{A}-T_{\text {sky }}\right) / \eta$. Since this calibration is performed directly at the antenna plane, any fluctuation of the front-end loss is directly translated into variations in $T_{\mathrm{NA}}$. To visualize this effect, $T_{\mathrm{NA}}$ computed by assuming constants $L_{1}$ and $L_{2}$ (see Table II) are plot in Fig. 16 superimposed to the $L_{2}$ long-term trend of Fig. 15 (lines labeled " $\propto L 1 L 2$ "). The attenuator values are rescaled to match the range of $T_{\mathrm{NA}}$. It is worth to recall that $L_{2}$ and $T_{\mathrm{NA}}$ in these plots are computed for the same external maneuvers but in a totally independent manner and from different raw data. However, in all cases, they follow each other almost perfectly. These plots justify the decision of using only NIR_CA for nominal operations.

However, using long series of data over the ocean, it has been observed that the small temporal variations of $T_{\mathrm{NA}}$ for NIR_CA are still translated into ripples in science measurements instead of stabilizing them. This fact suggests that the actual
NIR behavior is more stable than the externally calibrated $T_{\mathrm{NA}}$ suggests. The fact that the NIR is calibrated with the instrument looking to the sky, with different thermal conditions with respect to the nominal attitude, may contribute to this discrepancy.

\section{Calibration in Nominal Attitude}

In order to investigate this possibility, a calibration method using land-sea transitions with the instrument oriented at the nominal attitude was proposed in [26]. During a land-sea transition, the antenna temperature suffers a rapid change, which can be used as a two-level target to recalibrate the NIR. For a given land-sea transect, $T_{\mathrm{NA}}$ can be easily solved from (12) as

$$
T_{\mathrm{NA}}=-\frac{T_{\text {land }}-T_{\text {sea }}-\Delta T_{U}}{\eta_{\text {land }}-\eta_{\text {sea }}}
$$

where $\Delta T_{U}=T_{U \text { land }}-T_{U \text { sea }}$ and $T_{\text {land }}-T_{\text {sea }}$ is the antenna temperature jump between land and sea. It is independently estimated from LICEF PMS measurements using (1), where linearity and heater corrections (see Sections II-C and II-D) are applied. A total of 66 different values are obtained, one from each LICEF, but only 51 of them are kept after discarding those that show larger instabilities (see Fig. 11). The average of these ones is used in (14). For example, in [26], using a descending orbit passing over the coast of Africa, it was obtained that $T_{\text {land }}-T_{\text {sea }}=133.55 \mathrm{~K}$ for X-polarization and $124.89 \mathrm{~K}$ for Y-polarization, which introduced into (14) provided consistent $T_{\mathrm{NA}}$ values for all NIRs, similar to those obtained from external calibration (see [26] for details). Nevertheless, when using this calibration to measure the antenna temperature in the land or ocean zone, a difference with respect to LICEF measurements is still observed. For NIR_CA, it is about $2.4 \mathrm{~K}$ for X-polarization and $1.8 \mathrm{~K}$ for Y-polarization. Since gains are matched, this difference should be originated in the offset terms, either in NIR or LICEF or both. The fact that this mismatch is different for each NIR suggests that there is indeed offset errors in NIR.

The procedure was carried out for 447 orbits ranging from 2010 to 2017 [26]. Results are reproduced in Fig. 17 showing $T_{\mathrm{NA}}$ values retrieved in the nominal and external satellite orientations. Calibration using land-sea transitions is much noisier because of less data averaged for each retrieval, so a filtered version with a running average of 13 samples is added on top.

Absolute values are quite consistent in all NIR units, with a general tendency to be lower for the land-sea calibration 

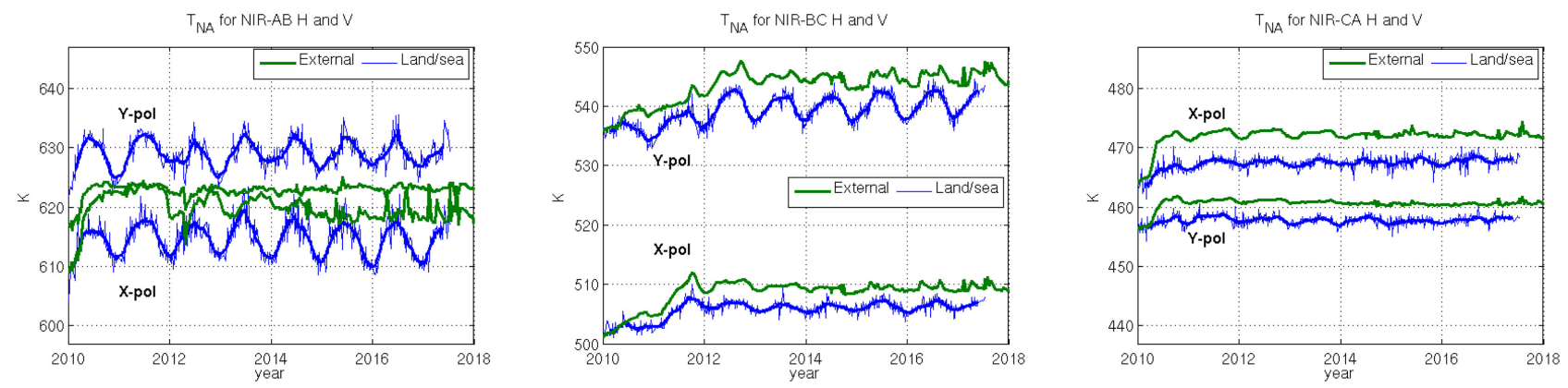

Fig. 17. NIR gain $\left(T_{\mathrm{NA}}\right)$ calibrated using land-sea transition (blue) and sky (green). Labels "x-pol” refer to units named "_H” and "y-pol” to those "_V."
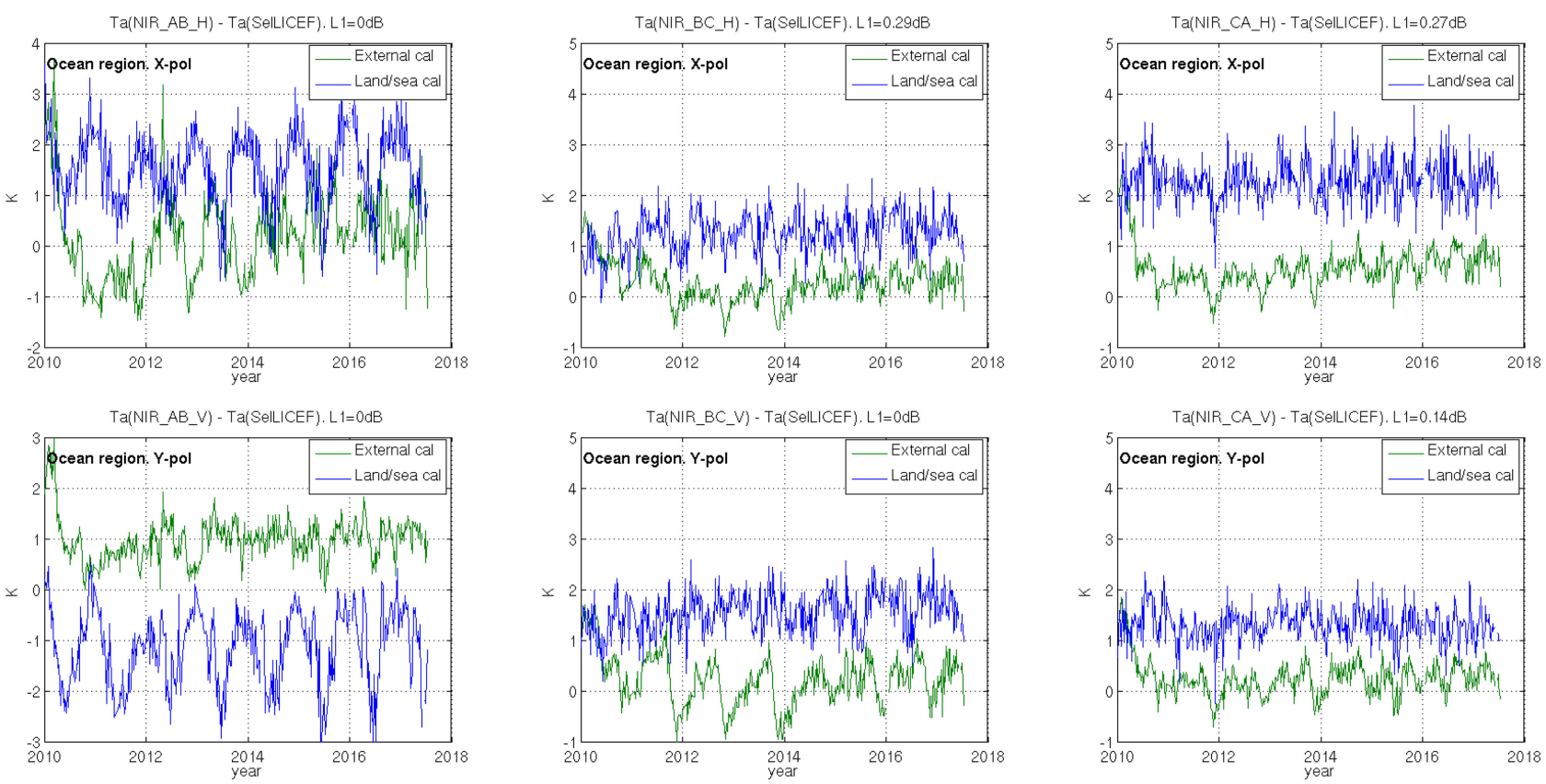

Fig. 18. Difference between NIR and LICEF antenna temperatures. NIR is calibrated by land-sea transitions.

(except for NIR_AB_V). More important is the different temporal variations observed in $T_{\mathrm{NA}}$ depending of the calibration approach. It should be recalled that the term depending on LICEF ( $\left.T_{\text {land }}-T_{\text {sea }}\right)$ in (14) is the same for all units so any variability is originated in the corresponding NIR. Since, as pointed out in Section II-C, $T_{\mathrm{NA}}$ temporal variations reflect changes in frontend loss, this result may indicate that NIR front-end loss change when the instrument rotates upwards to calibrate the NIR with respect to nominal attitude. And this change is different in each NIR unit.

NIR_AB shows a strong seasonal dependence, clearly not seen in sky-calibrated data. This might suggest a possible malfunction of the antenna connection, making it more sensitive to temperature variations. But this is not confirmed by other metrics.

NIR_BC shows a common initial trend, increasing up to 2012-2013, and then, stabilizing. However, NIR_BC_V is affected by strongest seasonal variations, as the ones seen in NIR_AB although somewhat smaller. They are present also in NIR_BC_H but to a lesser scale. Compared to NIR_AB, more consistent patterns between external and land-sea calibrations are seen.

NIR_CA is the most stable instrument. In this case, the landsea calibrated $T_{\mathrm{NA}}$ appears to be a little more stable than the sky calibrated one, especially in X-polarization (NIR_CA_H), but not dramatically. Some ripples in the sky calibration, especially in 2010-2013 are reproduced in a somewhat less amplitude in the land-sea calibrated plot.

Fig. 18 shows the difference between NIR and LICEF antenna temperatures in the ocean region. Both results, for NIR external and land/sea calibration, are shown. Since LICEF antenna temperature is the same in all cases, any different behavior must be attributed solely to the corresponding NIR.

Again NIR_AB shows a strong seasonal variability, which is not fully understood and points to possible erratic malfunctioning of the instrument. In this case, calibration using land/sea clearly does not stabilize the data, but produces more ripples.

On the other hand, NIR_BC does improve when calibrating using the land/sea approach. In this case, the relatively large 
ripples of $T_{\mathrm{NA}}$ seen in Fig. 17 correct the measurements and provide more stable results. This is especially true for Ypolarization, although it is seen in both.

Similar stable results are seen in NIR_CA, yet not so clear due to the inherent higher stability of this instrument. Taking apart the larger noise, results using land/sea calibration tend to be more stable than those using the nominal external calibration, especially around 2010. On the other hand, absolute values increase about 1 to $2 \mathrm{~K}$, which has an important impact on the SMOS brightness temperature absolute calibration. This fact and the larger thermal noise prevents yet from implementing this strategy in the nominal processor, avoiding abrupt changes in the calibration operations of the instrument.

\section{CONCLUSION}

Careful revision of the MIRAS power measurement system calibration shows that there is still margin for improvement. The gain long-term drift is stabilized by using a one-point approach based on a load at ambient temperature and the ground characterized receiver noise temperature. Nonlinearity is removed thanks to a clever use of the on ground diode characteristics. Erratic jumps in the instrumental offset are efficiently corrected by moving the effective date of the calibration data closer to the jump event, accurately estimated from consecutive orbits over the ocean. Finally, interference of the internal heater signal is compensated by using exponential models for both gain and offset.

Front-end loss, not calibrated internally, is accurately characterized by the ratio of external to internal calibration taking advantage of the external maneuvers carried out every two weeks. Results show long-term ripples in all antennas, with quite different behavior among them, although all of them having an increase in the first six months of the mission.

The NIR antenna temperature shows a clear dependence with the outer temperature of the antenna patch, minimized by an optimal value found for the antenna loss. NIR calibration parameters retrieved from standard external looks present long-term instabilities similar to those of total front-end loss, independently measured. Alternative NIR calibration using land-sea transitions in nominal pointing seems to indicate that the frontend loss behave differently depending on the attitude of the satellite.

\section{REFERENCES}

[1] M. Martín-Neira and J. M. Goutoule, "MIRAS - A two-dimensional aperture-synthesis radiometer for soil moisture and ocean salinity observations," ESA Bull., vol. 92, pp. 95-104, Nov. 1997.

[2] H. Barr, B. Duesmann, and Y. Kerr, "SMOS: The mission and the system," IEEE Trans. Geosci. Remote Sens., vol. 46, no. 3, pp. 587-593, Mar. 2008.

[3] K. McMullan et al., "SMOS: The payload," IEEE Trans. Geosci. Remote Sens., vol. 46, no. 3, pp. 594-605, Mar. 2008.

[4] M. Martín-Neira et al., "SMOS instrument performance and calibration after six years in orbit," Remote Sens. Environ., vol. 180, pp. 19-39, Feb. 2016.

[5] M. Martín-Neira, S. Ribó, and A. J. Martín-Polegre, "Polarimetric mode of MIRAS," IEEE Trans. Geosci. Remote Sens., vol. 40, no. 8, pp. 17551768, Aug. 2002.
[6] C. S. Ruf, C. T. Swift, A. B. Tanner, and D. M. Le Vine, "Interferometric synthetic aperture microwave radiometry for the remote sensing of the Earth," IEEE Trans. Geosci. Remote Sens., vol. 26, no. 5, pp. 597-611, Sep. 1988.

[7] I. Corbella, N. Duffo, M. Vall-llossera, A. Camps, and F. Torres, "The visibility function in interferometric aperture synthesis radiometry," IEEE Trans. Geosc. Remote Sens., vol. 42, no. 8, pp. 1677-1682, Aug. 2004.

[8] I. Corbella et al., "MIRAS end-to-end calibration. Application to SMOS L1 processor," IEEE Trans. Geosci. Remote Sens., vol. 43, no. 5, pp. 1126 1134, May 2005.

[9] F. Torres et al., "Denormalization of visibilities for in-orbit calibration of interferometric radiometers," IEEE Trans. Geosci. Remote Sens., vol. 44, no. 10, pp. 2679-2686, Oct. 2006.

[10] A. Colliander, M. Martín-Neira, J. Closa, and J. Benito, "Pre-launch estimation of radiometric resolution and stability of SMOS zero-baseline radiometer in anechoic chamber," IEEE Trans. Geosci. Remote Sens., vol. 48, no. 3, pp. 1389-1397, Mar. 2010.

[11] I. Corbella, V. Gonzlez-Gambau, F. Torres, N. Duffo, I. Duran, and M. Martín-Neira, "The MIRAS "All-LICEF" calibration mode," in Proc. IEEE Int. Geosci. Remote Sens. Symp., Jul. 10-15, 2016, pp. 2013-2016.

[12] F. Torres, A. Camps, J. Bará, I. Corbella, and R. Ferrero, "On-board phase and modulus calibration of large aperture synthesis radiometers: Study applied to MIRAS," IEEE Trans. Geosci. Remote Sens., vol. 34, no. 4, pp. 1000-1009, Jul. 1996.

[13] J. Lemmetyinen et al., "SMOS calibration subsystem," IEEE Trans. Geosci. Remote Sens., vol. 45, no. 11, pp. 3691-3700, Nov. 2007.

[14] A. Colliander et al., "Development and calibration of SMOS reference radiometer," IEEE Trans. Geosci. Remote Sens., vol. 45, no. 7, pp. 19671977, Jul. 2007.

[15] J. Kainulainen, J. Lemmetyinen, K. Rautiainen, A. Colliander, J. Uusitalo, and J. Lahtinen, "Error propagation in calibration networks of synthetic aperture radiometers," IEEE Trans. Geosci. Remote Sens., vol. 47, no. 9, pp. 3140-3150, Sep. 2009.

[16] M. Brown, F. Torres, I. Corbella, and A. Colliander, "SMOS calibration," IEEE Trans. Geosci. Remote Sens., vol. 46, no. 3, pp. 646-658, Mar. 2008.

[17] P. Piironen, "PMS offset determination using an IF attenuator," ESAESTEC, Noordwijk, The Netherlands, Tech. Note 14629/00/NL/SF, Jun. 2002.

[18] F. Torres, N. Duffo, I. Corbella, A. Camps, M. Vall-1lossera, and L. Sagués, "Dynamic range and linearity tradeoff in detectors for interferometric radiometers," IEE Electron. Lett., vol. 39, no. 25, pp. 1852-1854, Dec. 11, 2003.

[19] C. Gonzalez-Haro et al., "Linearity characterization of detectors for interferometric radiometers," in Proc. IGARSS Int. Geosci. Remote Sens. Symp., vol. 4, Jul. 12-17, 2009, pp. 657-660.

[20] W. J. Wilson, A. B. Tanner, F. A. Pellerano, and K. A. Horgan, "Ultra stable microwave radiometers for future sea surface salinity missions," Jet Propulsion Lab., California Inst. of Technol., Pasadena, CA, USA, Apr. 2005. [Online]. Available: https://ntrs.nasa.gov/search. jsp? $\mathrm{R}=20080025042$

[21] I. Corbella et al., "MIRAS calibration and performance. Results from the SMOS in-orbit commissioning phase," IEEE Trans. Geosci. Remote Sens., vol. 49, no. 9, pp. 3147-3155, Sep. 2011.

[22] I. Corbella et al., "On-ground characterization of the SMOS payload," IEEE Trans. Geosci. Remote Sens., vol. 47, no. 9, pp. 3123-3133, Sep. 2009.

[23] I. Corbella, F. Torres, N. Duffo, I. Durn, M. Pablos, and M. MartínNeira, "Enhanced SMOS amplitude calibration using external target," in Proc. IEEE Int. Geosci. Remote Sens. Symp., Jul. 22-27, 2012, pp. 2868-2871.

[24] D. M. Le Vine, S. Abraham, Y. H. Kerr, W. J. Wilson, N. Skou, and S. S. Sobjaerg, "Comparison of model prediction with measurements of galactic background noise at L-band," IEEE Trans. Geosci. Remote Sens., vol. 43, no. 9, pp. 2018-2023, Sep. 2005.

[25] J. Kainulainen et al., "Radiometric performance of the SMOS reference radiometers-Assessment after one year of operation," IEEE Trans. Geosci. Remote Sens., vol. 50, no. 5, pp. 1367-1383, May 2012.

[26] I. Corbella, F. Torres, N. Duffo, I. Duran, R. Oliva, and M. Martín-Neira, "Self-consistent amplitude calibration of MIRAS-SMOS," in Proc. 15th Spec. Meeting Microw. Radiometry Remote Sens. Environ., Boston, MA, USA, Mar. 27-30, 2018, pp. 116-120. 


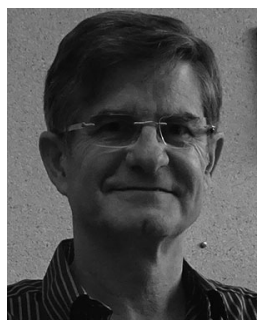

Ignasi Corbella was born in Barcelona, Spain, in 1955. He received the Telecommunication Engineering degree and Doctoral degree in telecommunication engineering from the Universitat Politcnica de Catalunya (UPC), Barcelona, in 1977 and 1983, respectively.

In 1976, he was with the Microwave Laboratory, School of Telecommunication Engineering, UPC, as a Research Assistant, where he worked on passive microwave integrated-circuit design and characterization. In 1979, he was with Thomson-CSF, Paris, France, working on microwave oscillators design. In 1982, he was an Assistant Professor, in 1986, an Associate Professor, and in 1993, became a Full Professor with the UPC, where he is currently teaching basic microwaves and antennas at undergraduate level and graduate courses on nonlinear microwave circuits. Since 1993, he has been actively participating as a Researcher with the European Space Agency (ESA) Soil Moisture and Ocean Salinity (SMOS) mission in the frame of several contracts, directly with the ESA, with the payload prime contractor EADS-Casa Espacio, Madrid, Spain, or with operational processor prime contractor Deimos Engenharia, Lisbon, Portugal. His expertise includes, among others, fundamentals of interferometric aperture synthesis radiometry, radiometer calibration, image reconstruction, radiometer hardware specification, and payload characterization. From 1993 to 1997, he was the Academic Director with the School of Telecommunications Engineering, and from 2001 to 2003, he was the Director with the Department of Signal Theory and Communications, UPC. From 1998 to 1999, he was a Guest Researcher with the NOAA/Environmental Technology Laboratory, Boulder, CO, USA, developing methods for total-power radiometer calibration and data analysis. In 2015, he was a Visiting Scholar with the University of Colorado at Boulder, Boulder, working on emission models from layered media. From 2004 to 2010, he was a Member of the SMOS Science Advisory Group, and since 2010, the SMOS Quality Working Group. He was the Scientific Coordinator of a Dictionary of Telecommunication terms in Catalan language, with more than 4000 entries, published in March 2007.

Dr. Corbella was the General Chairman of the 2007 International Geoscience and Remote Sensing Symposium, Barcelona.

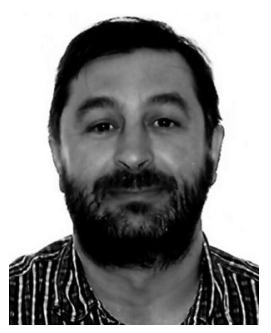

Francesc Torres (S'88-M'96-SM'06) was born in Ibiza, Spain, in 1962. He received the degree in telecommunications engineering from the Barcelona School of Telecommunications Engineering (ETSETB), Universitat Politcnica de Catalunya (UPCBarcelonaTech), Barcelona, Spain, in 1988.

$\mathrm{He}$ is a Full Professor with the UPC, where he teaches and does research in the field of radio communications and Earth observation. He started his professional career with the European Space Agency (ESA), Holland, where he worked from 1988 to 1989. He has been the Head of studies with the ETSETB during 1998-2000, a Coordinator of the MERIT Erasmus Mundus international research master's degree, during 2006-2009, and the Director with the Department of Signal Theory and Communications, UPC, during 2013-2017. He is currently the Rector with the UPC-BarcelonaTech. Through the Remote Sensing Laboratory (RSLab-UPC), he has participated in more than 70 contracts funded by government bodies and industry, mainly for the development of the ESAs Soil Moisture and Ocean Salinity (SMOS) explorer, the first satellite led by Spanish industry (EADSCASA Espacio), which has provided data for studying the water cycle and improving climate models since 2009. He is a Member of the UPC's Mara de Maeztu Unit CommSensLab. He has authored more than 200 papers published in prestigious international journals and presented at international conferences.

Prof. Torres was the recipient of the Award for Improving Quality in University Teaching, in 1997, from the UPC's Board of Trustees for the Radiation and Guided Wave Laboratory, ETSETB. He has also received several recognitions as a Member of the RSLab, including, the Duran Farell Prize of the Board of Trustees in 2000, the City of Barcelona Prize in 2001, the prize of the Catalan Association of Telecommunications Engineers in 2004, and the Aeronautics Prize of Barcelona City Council in 2011. He was the Founding Vice-Chair of the SMOS Barcelona Expert Centre in 2007-2010 and also had a sabbatical year as an External Consultant of the GeoSTAR project with NASA's Jet Propulsion Laboratory, Caltech, CA, USA, in 2005-2006.

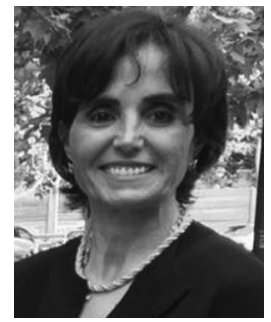

Nuria Duffo (S'91-M'95-SM'13) received the Telecommunication Engineering degree and the $\mathrm{Ph} . \mathrm{D}$. degree in telecommunication engineering from the School of Telecommunications Engineering, Universitat Politcnica de Catalunya (UPC), Barcelona, Spain, in 1990 and 1996, respectively.

Since 1997, she has been an Associate Professor with the Department of Signal Theory and Communications, UPC. Her current research interests include numerical methods in electromagnetics, microwave radiometry, antenna analysis, and design.

Dr. Duffo was the part of the organizing committee of the International Geoscience and Remote Sensing Symposium, Barcelona, July 22-27, 2007.

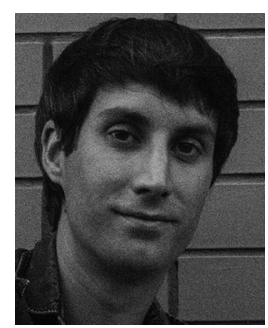

Israel Durán was born in Esplugues de Llobregat, Spain. He received the Telecommunication Engineering and Dr. Eng. degrees in Telecommunication Engineering from the Universitat Politècnica de Catalunya (UPC), Barcelona, Spain, in 2010 and 2017, respectively.

$\mathrm{He}$ is currently with the Passive Remote Sensing Group, Signal Theory and Communications Department, UPC. His research activities are related to SMOS mission data processing.

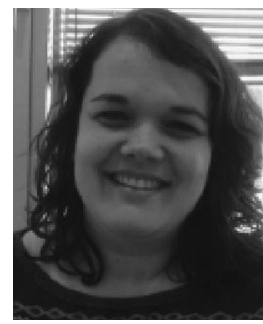

Verónica González-Gambau was born in Huesca, Spain, in 1981. She received the M.S. and Ph.D. degrees in telecommunication engineering from the School of Telecommunication Engineering, Universitat Politcnica de Catalunya (UPC), Barcelona, Spain, in 2006 and 2012, respectively.

In 2006, she joined the Passive Remote Sensing Group, Signal Theory and Communications Department, UPC, where she was involved in the Soil Moisture and Ocean Salinity (SMOS) payload onground characterization in the framework of SMOS precommissioning activities. She was also collaborating with the SMOS Level 1 software development and data analysis of the Microwave Imaging Radiometer by Aperture Synthesis instrument validation campaigns. In 2007, she was a UPC Researcher in collaboration with the Barcelona Expert Center, Barcelona. She is currently a Research Scientist with the Institut de Ciéncies del MarConsejo Superior de Investigaciones Científicas, Barcelona, the SMOS mission coleading institution, where she is responsible for the advance Level 1 error correction techniques and in-orbit validation procedures. She is working on the development of algorithms for the SMOS brightness temperatures improvement and assessing the impact of these techniques on the quality of the geophysical retrievals.

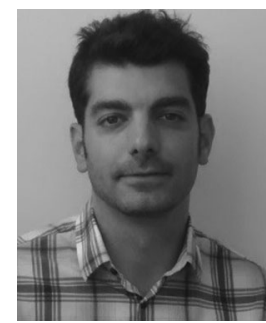

Roger Oliva (M'18) received the M.Sc. degree in telecommunication engineering from the Polytechnic University of Catalonia, Barcelona, Spain, in 2004.

He has worked in several space and astronomy projects, including the European Space Agency's mission Mars Express, two astronomical microwave observatories and in the design of advanced telecommunications satellite payloads. Since 2007, he has been working with the European Space Agency Earth Observation satellite Soil Moisture and Ocean Salinity (SMOS). In SMOS, he is the Coordinator of the Calibration and Level 1 Processing Team and leads the SMOS Radio Frequency Interference Team.

Mr. Oliva was the recipient of the Certificate of Appreciation from the IEEE Geoscience and Remote Sensing Society's Frequency Allocations in Remote Sensing (FARS) Committee for their successful efforts in working with national authorities in removing radio-frequency interference sources from the protected 14000-1427 MHz EESS band, in 2013. Since 2017, he has been a Co-chair of the FARS Technical Committee. 


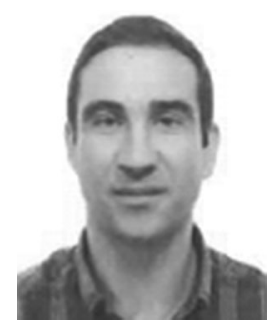

Josep Closa received the degree in electronic engineering from the Polytechnic University of Catalonia, Barcelona, Spain, in 1997.

He started his career with ESA-ESRIN working for the ENVISAT Ground Segment Department as an Advanced Synthetic Aperture Radar (ASAR) Processor and Products Development Engineer. He is currently with the Microwave Instruments Department, Airbus Defence and Space, Madrid, Spain . He has almost 20 years of professional experience in active and passive SAR and radiometers mission development and the associated performances, in-flight calibration activities such as the ones for the ENVISAT-ASAR, PAZ (X-band SAR), and the Soil Moisture and Ocean Salinity (SMOS).

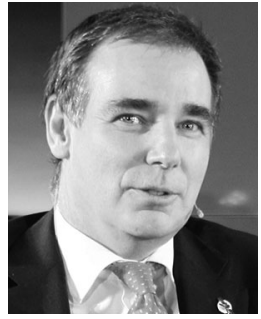

Manuel Martín-Neira (M'96-SM'07) received the M.S. and Ph.D. degrees in telecommunication engineering from the School of Telecommunication Engineering, Polytechnic University of Catalonia, Barcelona, Spain, in 1986 and 1996, respectively.

In 1988, he was awarded a fellowship to work on microwave radiometry with the European Space Agency (ESA), Noordwijk, Netherlands. From 1989 to 1992 , he was with GMV, a Spanish firm, as a responsible for several projects on GPS spacecraft precise navigation and attitude determination. Since 1992, he has been an in charge of the radiometer activities within the Payload, Equipment and Technology Section, ESA. In 2010, he became a Remote Sensing Senior Engineer with the Payload Systems Division, ESA. Since 2001, he has been the Instrument Principal Engineer with ESAs Soil Moisture and Ocean Salinity (SMOS) mission. He has developed new concepts for constellations of small satellites for Earth Observation. In particular, he holds several patents related to aperture synthesis radiometry and on the use of GNSS signals reflected from the ocean (PARIS concept).

Dr. Martín-Neira was the recipient of the Confirmed Inventor Award from the ESA in 2002, the Salva i Campillo Award and the Premio Jaime I in 2010 from Spain, and a Certificate of Recognition for the SMOS mission in 2011 from the IEEE. He is member of the Academie des Technologies of France. Since 2008, he is working in potential GNSS reflectometry missions like the PARIS In-orbit Demonstration mission, GEROS-ISS experiment on the International Space Station, and the PRETTY GNSS-R cubesat. More recently, he has proposed a system to perform Space Very Long Baseline Interferometry from Medium Earth Orbit to image the event horizon of Super Massive Black Holes like Sagittarius A* or M87. 